\title{
Acúmulo, deposição e decomposição de serrapilheira sob a dinâmica vegetacional da Caatinga em Unidade de Conservação
}

\section{Accumulation, deposition and decomposition of litter in a dynamic Caatinga vegetation in a Conservation Unit}

\author{
Íkallo George Nunes Henriques ${ }^{*}$, Jacob Silva Souto ${ }^{2}$, Patrícia Carneiro Souto ${ }^{3}$, William de Sousa Santos ${ }^{4}$, Ingrid Gisely \\ Nunes Henriques ${ }^{5}$, Tiago Silva Lima ${ }^{6}$
}

Resumo: A ciclagem de nutrientes é essencial para a manutenção da produtividade dos ecossistemas florestais, principalmente sobre solos de baixa fertilidade e fortemente intemperizados. Apesar da existência de trabalhos de acúmulo e deposição de serrapilheira da vegetação da Caatinga, ainda falta muito para o conhecimento da dinâmica desse bioma. O presente trabalho teve por objetivo, avaliar o acúmulo, deposição e decomposição de serrapilheira, a fim de obter informações sobre a dinâmica das espécies vegetais de uma Reserva Particular do Patrimônio Natural no bioma Caatinga, no Estado da Paraíba, para propiciar futuros estudos no tocante a ciclagem de nutrientes e, avaliar a interferência da precipitação pluviométrica na sazonalidade destes eventos. Para se obter a produção de serrapilheira 20 coletores de $1,0 \mathrm{~m} \mathrm{x} \mathrm{1,0} \mathrm{m,} \mathrm{com} \mathrm{fundo} \mathrm{da} \mathrm{tela} \mathrm{de}$ náilon. Coletada mensalmente, a serrapilheira foi separada nas frações folhas, galhos, material reprodutivo e miscelânea, sendo as frações secas em estufa e posteriormente pesada. Para avaliar a quantificação do estoque de serrapilheira acumulada foi utilizada moldura metálica com dimensões de $0,5 \mathrm{~m}$ x $0,5 \mathrm{~m}$, lançada aleatoriamente, sendo coletada mensalmente, levada ao laboratório para secagem em estufa e pesado. A deposição de serrapilheira na fração folha no ano de 2011 estimou-se em $2.079,61 \mathrm{~kg} \mathrm{ha}^{-1}$, representando $77,23 \%$ do total estimado para o período experimental. Conclui-se que, a produção de serrapilheira obedeceu a seguinte ordem: folhas > galhos + cascas > material reprodutivo > miscelânea. A serrapilheira acumulada no piso florestal na área de estudo pode ser decomposta em quase sua totalidade em aproximadamente dois anos.

Palavras-chave: Aporte de serrapilheira. Ciclagem de nutrientes. Miscelânea.

\begin{abstract}
Nutrient cycling is essential for maintaining the productivity of forest ecosystems, especially on soils of low fertility and strongly weathered. Despite the studies of accumulation and deposition of litter in the Caatinga vegetation, there is still much to our understanding of the dynamics of this biome. This study aimed to evaluate the accumulation, deposition and decomposition of litter in order to obtain information about the dynamics of plant species in a Private Reserve of Natural Heritage in Caatinga biome in the state of Paraíba, to provide future studies regarding nutrient cycling, to facilitate future studies regarding nutrient cycling, and evaluate the role of rainfall seasonality in these events. To obtain the litterfall production, we used 20 collectors of $1.0 \mathrm{~m}$ x $1.0 \mathrm{~m}$, with nylon fabric background. The collection was done monthly, the litter was separated into leaves, twigs, reproductive material and miscellaneous and were dried in an oven and then weighed. To evaluate the quantification of the stock of accumulated litter, we used a metal frame with dimensions of $0.5 \mathrm{~m} \times 0.5 \mathrm{~m}$, thrown randomly being and being collected monthly, then taken to the laboratory oven to dry and to be weighed. The deposition fraction of leaf litter in the year 2011 was estimated at $2079.61 \mathrm{~kg} \mathrm{ha}^{-1}$, representing $77.23 \%$ of the total estimate for the experimental period. We conclude that the litterfall followed the order: leaves $>$ bark + branches $>$ reproductive material $>$ miscellany. The litter accumulated on the forest floor in the study area can be decomposed almost completely in about two years.
\end{abstract}

Key words: Contribution of litterfall. Nutrient cycling. Miscellaneous

\footnotetext{
* Autor para correspondência

Recebido para publicação em 12/11/2015; aprovado em 10/02/2016

${ }^{1}$ Engenheiro Florestal, CSTR/UFCG, Patos, Paraíba, Brasil. Fone (83) 9 9652-7373. E-mail: ikallogeorge@ yahoo.com.br

${ }^{2}$ Professor Titular da Unidade Acadêmica de Engenharia Florestal/CSTR/PPGCF/UFCG, Patos-PB; E-mail: jacob_souto@yahoo.com.br

${ }^{3}$ Professora da Unidade Acadêmica de Engenharia Florestal/CSTR/PPGCF/UFCG, Patos-PB; E-mail: pcarneirosouto@yahoo.com.br

${ }^{4}$ Mestrando no PPGCF/CSTR/UFCG, Patos - PB; E-mail: william.ufcg@gmail.com

${ }^{5}$ Mestranda no PPGCF/CSTR/UFCG, Patos - PB; E-mail: ingrid_gisely@ hotmail.com

${ }^{6}$ Estudantes do curso de Agronomia da Universidade Federal de Campina Grande, Campus Pombal-PB, E-mail: lima_tiago92@ outlook.com
} 


\section{INTRODUÇÃO}

O desenvolvimento do Brasil é marcado por uma intensa exploração do meio ambiente. O progresso levou a uma crescente demanda de recursos, induzindo o homem a explorar cada vez mais a natureza, sendo que, na maioria das vezes, sem um planejamento adequado, resultando em uma rápida degradação do meio.

A Caatinga é um bioma brasileiro muito explorado nos dias atuais, pela sua fonte de energia, sendo a madeira o principal recurso explorado, o que pode degradar cada vez mais essa região semiárida.

A vegetação da Caatinga apresenta, em sua maioria, espécies caducifólias que perdem toda ou parcialmente a folhagem como mecanismo fisiológico de adaptação aos períodos prolongados de estiagem e déficit hídrico no solo. Essa deposição de material orgânico na superfície do solo promove a formação de uma camada de resíduos denominada serrapilheira, que vai ser decomposta pelos organismos do solo, sendo de grande importância para a ciclagem de nutrientes e a manutenção da umidade e atividade biológica da área.

A serrapilheira pode ser chamada de camada orgânica superficial do solo de ecossistemas florestais, sendo formada por folhas, galhos, material reprodutivo e miscelânea (COSTA et al., 2010).

Na região Nordeste do Brasil, particularmente em área de caatinga, pouco se conhece sobre as taxas de deposição e acúmulo das espécies florestais nativas. A serrapilheira que cai das árvores nesse ecossistema protege o solo na estação seca quando as temperaturas são mais elevadas, mas, logo que chegam as primeiras chuvas, ela é degradada pelos microrganismos decompositores, não ocorrendo grande acúmulo de material orgânico na superfície (SOUTO, 2006).

É premente a necessidade de estudos sobre a vegetação e a serrapilheira, principalmente sobre os efeitos desta na conservação do solo. Por isso, um estudo detalhado sobre a produção da serrapilheira é importante na busca de informações sobre a ciclagem dos nutrientes em área de caatinga, conhecendo a quantidade produzida e decomposta, sazonalmente, por essas áreas.

Estudos sobre a dinâmica da vegetação nativa estão sendo desenvolvidos em Reservas Particulares do Patrimônio Natural (RPPN), pois fornecem condições para coleta de dados mais consistentes, pelos fatores de ausência de atividades antrópicas e a conservação e o equilíbrio em que se encontram essas áreas, em um tempo prolongado. Passando aos pesquisadores, informações de como seriam o desempenho das espécies e dos fatores abióticos e bióticos na sua formação e manutenção (PAZ, 2010).

O objetivo desse estudo foi avaliar o acúmulo, deposição e decomposição de serrapilheira, a fim de obter informações sobre a dinâmica das espécies vegetais de uma RPPN (Reserva Particular do Patrimônio Natural) no bioma Caatinga, Estado da Paraíba, para propiciar futuros estudos no tocante à ciclagem de nutrientes e, avaliar a interferência da precipitação pluviométrica na sazonalidade destes eventos.

\section{MATERIAL E MÉTODOS}

\section{Caracterização da área de estudo}

O experimento foi conduzido na RPPN Fazenda Tamanduá, situada no município de Santa Terezinha (PB), na
Mesorregião do Sertão Paraibano, distante $18 \mathrm{~km}$ da cidade de Patos (Figura 1).

Figura 1 - Mapa da Paraíba e localização do município de Santa Terezinha onde se encontra a RPPN da Fazenda Tamanduá.
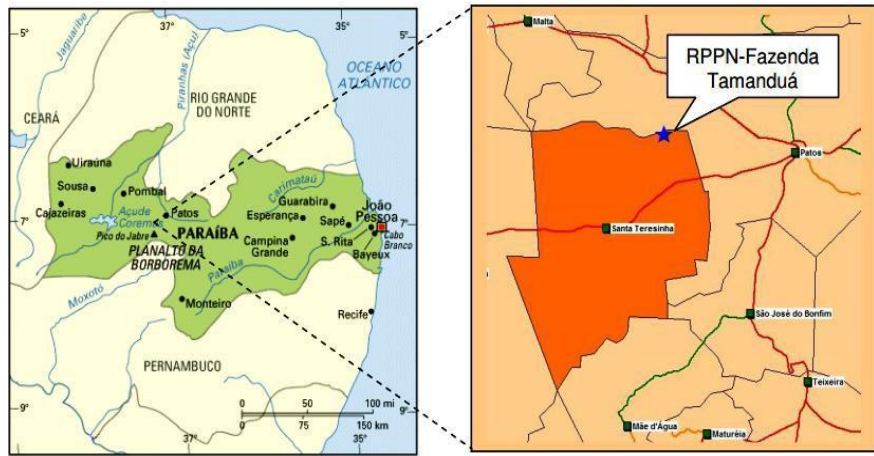

Fonte: Adaptado de Araújo (2007).

A RPPN possui uma área de 325 ha que não é explorada há quase 50 anos, sendo a vegetação caracterizada como Caatinga arbustiva-arbórea fechada (SOUTO et al. 2009; SOUTO et al. 2013).

Os solos predominantes na área experimental são do tipo NEOSSOLOS LITÓLICOS (EMBRAPA, 2006), com afloramentos de rochas e topografia com fortes ondulações, apresentando serrotes.

O clima onde se encontra a área experimental, caracterizase como sendo do tipo BSh, semiárido quente de tendência tropical, segundo Koppen, com precipitação inferior a 1000 $\mathrm{mm}$ ano-1 e temperatura média do ar superior a $25{ }^{\circ} \mathrm{C}$ (Koppen, 1996).

Quanto à vegetação da área, no entorno dos coletores (raio de $\pm 50,0 \mathrm{~m}$ ), amostrada por Souto (2006), constituída predominantemente pelo estrato arbóreo, sendo as espécies mais representativas como a Poincianella pyramidalis (Fabaceae), Croton blanchetianus (Euphorbiaceae), Combretum leprosum (Combretaceae), Anadenanthera colubrina (Mimosaceae), Sida sp (Malvacea), Hyptis suaveolens Poit (Lameaceae), Piptadenia stipulacea (Mimosaceae), Commiphora leptophloeos (Burseraceae), Genipa americana (Rubiaceae), Pseudobombax $c f$. marginatum (Bombacaceae), Capparis flexuosa (Capparaceae), Aspidosperma pyrifolium (Apocynaceae), Mimosa tenuiflora (Mimosaceae), Bauhinia cheilantha (Fabaceae).

\section{Coleta de dados \\ Precipitação pluviométrica}

A precipitação pluviométrica na Fazenda Tamanduá foi registrada mensalmente, em uma miniestação climatológica, onde estão instalados pluviômetros.

\section{Aporte de serrapilheira}

Para avaliar a deposição de serrapilheira na RPPN, foi seguida a metodologia utilizada por Souto (2006), sendo utilizados 21 coletores, com dimensões de $1,0 \mathrm{~m} \times 1,0 \mathrm{~m}$, fundo de tela de náilon com malha de $1,0 \mathrm{~mm}^{2}$, instalados $20,0 \mathrm{~cm}$ acima da superfície do solo (Figura 2). 
Figura 2 - Caixas coletoras de serrapilheira na área experimental.

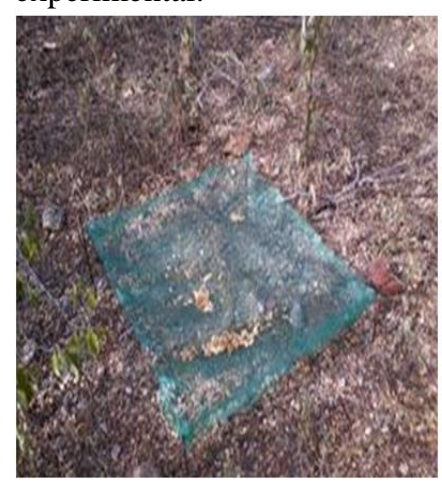

Fonte: Henriques (2012).

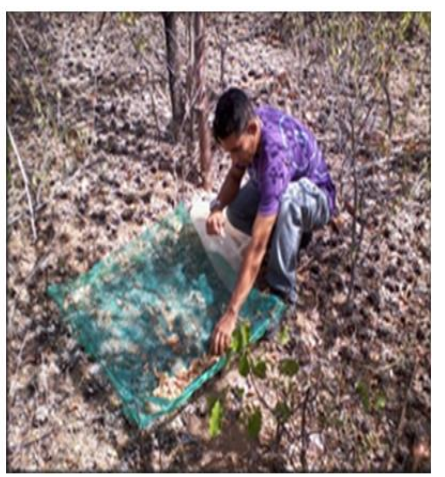

Em seguida, foram demarcados sete transectos com $200 \mathrm{~m}$ de distância entre si, sendo distribuídos, aleatoriamente, os coletores a $50 \mathrm{~m}$ metros da estrada, equidistantes cerca de 30 m.

As coletas foram realizadas durante um período de 21 meses, compreendido entre dezembro/2010 a agosto/2012. O material interceptado pelos coletores foi recolhido regularmente em intervalos de 30 dias, sendo acondicionado em sacos plásticos devidamente identificados, levados ao Laboratório de Nutrição Mineral de Plantas da Unidade Acadêmica de Engenharia Florestal da Universidade Federal de Campina Grande, campus de Patos-PB.

No laboratório, a serrapilheira coletada foi separada manualmente nas seguintes frações: folhas (incluindo folíolos + pecíolo), galhos (correspondente às partes lenhosas arbóreas de todas as dimensões + cascas), estruturas reprodutivas (flores, frutos e sementes), e miscelânea (material orgânico em estado avançado de decomposição, restos de animais).

Em seguida, cada fração de serrapilheira foi acondicionada em sacos de papel identificados e levados para secagem em estufa de circulação forçada de ar a $\pm 70{ }^{\circ} \mathrm{C}$ por 72 horas, sendo, então, pesados em balança de precisão $( \pm$ $0,01 \mathrm{~g})$.

\section{Acúmulo de serrapilheira no piso florestal}

$\mathrm{O}$ estoque de serrapilheira acumulada sobre a superfície do solo foi determinado através de coletas mensais, entre dezembro/2010 e agosto/2012, utilizando-se moldura metálica com dimensões de $0,5 \mathrm{~m}$ x $0,5 \mathrm{~m}$, lançada de forma aleatória (Figura 3).

Figura 3: Moldura metálica utilizada para coleta da serrapilheira acumulada.
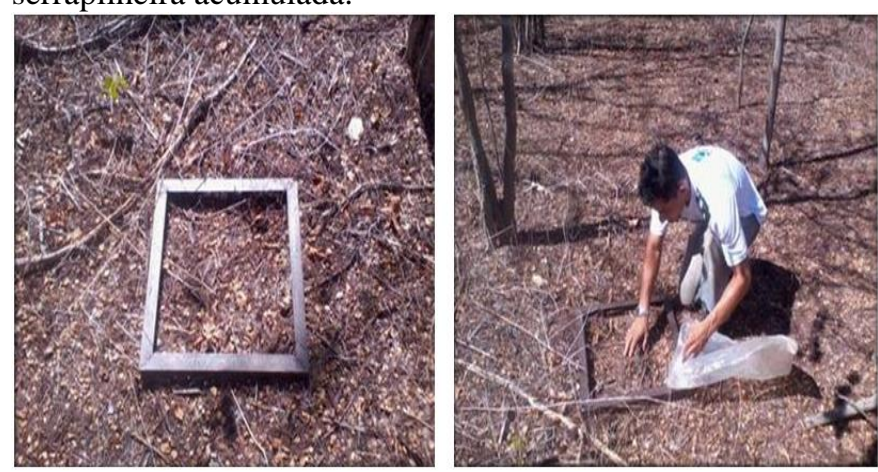

Fonte: Henriques (2012)
Foram coletadas três amostras em cada transecto, totalizando 21 amostras, próximas aos coletores de serrapilheira. Foi considerado como serrapilheira acumulada, todo material vegetal decíduo depositado sobre o solo, em diferentes graus de decomposição, dentro do espaço delimitado pela moldura. Após a coleta, as amostras foram acondicionadas em embalagens plásticas, devidamente identificadas, levadas ao laboratório, secas e pesadas em balança de precisão.

\section{Estimativa da taxa de decomposição da serrapilheira}

A taxa de decomposição da serrapilheira foi estimada através da equação abaixo proposta por Olson (1963), e empregada em estudos semelhantes (Queiroz, 1999; Vital, 2002; Kolm; Poggiani, 2003; Arato et al., 2003).

em que:

$$
\mathrm{K}=\mathrm{L} / \mathrm{Xss}
$$

$\mathrm{K}=$ constante de decomposição

$\mathrm{L}=$ produção anual de serrapilheira $\left(\mathrm{g} \mathrm{m}^{-2}\right)$

Xss = média anual da serrapilheira acumulada sobre o solo $(\mathrm{g}$ $\left.\mathrm{m}^{-2}\right)$.

$\mathrm{O}$ valor $\mathrm{K}$ ou taxa de decomposição instantânea é a relação massa de serrapilheira produzida/massa de serrapilheira acumulada (ANDERSON; INGRAM, 1989). A partir do valor de $\mathrm{K}$, calculou-se, também, o tempo médio de renovação estimado por $1 / \mathrm{K}$ e os tempos necessários para que ocorra decomposição de $50 \%$ (t $\quad 0,5)$ e $95 \%$ (t $\quad 0,05)$ da serrapilheira, estimados pela equação de Shanks e Olson (1961):

$$
\begin{gathered}
\mathrm{t}_{0,5}=\operatorname{In} 2 / \mathrm{K}=0,693 / \mathrm{K} \\
\mathrm{t}_{0,05}=3 / \mathrm{K}
\end{gathered}
$$

\section{RESULTADOS E DISCUSSÃO}

\section{Precipitação pluviométrica e produção de serrapilheira}

Durante o período experimental foram coletados os dados relativos à precipitação mensal, os quais se encontram na Figura 4.

Figura 4- Precipitação pluviométrica registrada na área de estudo entre o período de dezembro de 2010 a agosto de 2012.

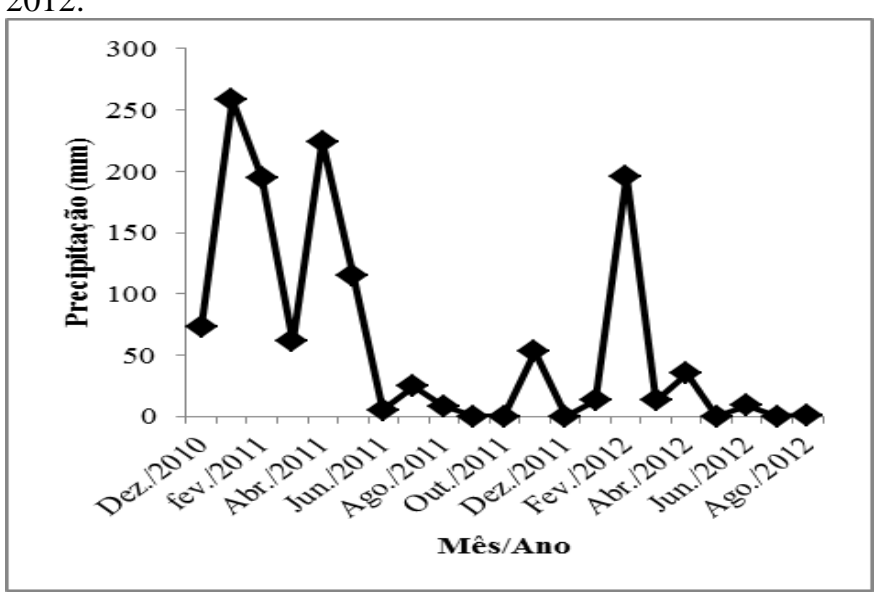

Nota-se que a distribuição da precipitação pluviométrica se apresentou muito irregular entre os anos, observando-se uma grande diminuição na precipitação a partir do mês de 
junho de 2011, contudo, para este mesmo ano a precipitação total atingiu valores elevados quando se compara com o resultado de anos anteriores. No entanto é possível se constatar uma má distribuição das chuvas durante o ano, ocorrendo uma concentração das precipitações $(90,34 \%)$ no período de janeiro a maio de 2011. Já em 2012, choveu apenas $257,2 \mathrm{~mm}$ no período de janeiro a maio, mostrando dessa forma a alta variabilidade das precipitações no semiárido da Paraíba.
Quanto à análise dos dados da serrapilheira sua totalidade foi de $4.207,84 \mathrm{~kg} \mathrm{ha}^{-1}$, houve diferenças de deposição por frações. À produção de folhas durante o período de estudo foi estimada em 2.692,69 $\mathrm{kg} \mathrm{ha}^{-1}$. No ano de 2011 estimou-se a produção de folhas em $2.079,61 \mathrm{~kg} \mathrm{ha}^{-1}$, representando $77,23 \%$ do total estimado para o período experimental, sendo que, $31,68 \%$ deste total foram obtidos no mês de agosto de 2011, período em que as precipitações pluviométricas foram da ordem de $8,2 \mathrm{~mm}$, estação seca da região (Tabela 1).

Tabela 1. Deposição das frações folha, galhos, estrutura reprodutiva e miscelânea em $\mathrm{kg} \mathrm{ha}^{-1}$ no período de dezembro de 2010 a agosto de 2012, Santa Terezinha, Paraíba.

\begin{tabular}{cccccc}
\hline Mês/Ano & Folha & Galhos + Cascas & M. Reprodutivo & Miscelânea & Total \\
\hline Dez./2010 & 29,77 & 46,41 & 17,89 & 0 & 94,07 \\
Jan./2011 & 49,69 & 46,66 & 43,3 & 0,01 & 139,66 \\
fev./2011 & 44,88 & 42,15 & 39,11 & 0,01 & 126,15 \\
Mar./2011 & 84,18 & 35,93 & 19,42 & 3,51 & 1,4 \\
Abr./2011 & 89,32 & 33,87 & 14,36 & 0,52 & 133,04 \\
Mai./2011 & 132,02 & 73,28 & 68,3 & 1,78 & 274,12 \\
Jun./2011 & 216,49 & 72,28 & 62,39 & 0,33 & 352,94 \\
Jul./2011 & 381,8 & 44,98 & 37,43 & 0 & 464,54 \\
Ago./2011 & 658,92 & 68,32 & 26,38 & 0 & 753,62 \\
Set./2011 & 212,79 & 42,02 & 24,45 & 0 & 279,26 \\
Out./2011 & 105,03 & 22,82 & 11,78 & 0 & 139,63 \\
Nov./2011 & 30,04 & 34,38 & 19,69 & 0 & 84,11 \\
Dez./2011 & 74,45 & 86,29 & 36,48 & 0,55 & 197,22 \\
Jan./2012 & 68,3 & 66 & 47,42 & 1,44 & 182,27 \\
Fev./2012 & 56,54 & 56,57 & 52,87 & 1,71 & 167,42 \\
Mar./2012 & 47,48 & 37,65 & 40,68 & 0,11 & 127,52 \\
Abr./2012 & 28,66 & 16,75 & 11,8 & 3,82 & 57,32 \\
Mai./2012 & 58,08 & 17,6 & 14,27 & 1,66 & 93,77 \\
Jun./2012 & 122,41 & 12,49 & 10,63 & 6,1 & 0,48 \\
Jul./2012 & 130,59 & 23,48 & 0,83 & 0,07 & 147,19 \\
Ago./2012 & 71,25 & 12,24 & 605,58 & 17,4 & 84,39 \\
\hline Total & $2.692,69$ & 892,17 & & $4.207,84$ \\
\hline
\end{tabular}

A produção mensal de serrapilheira total foi ininterrupta durante todo o período de coleta, mostrando, entretanto, marcante sazonalidade. A sazonalidade das variações na deposição da fração folhas permite inferir aos estudiosos no assunto que se tenha o conhecimento de como a vegetação responde às variações climáticas, a distribuição e estoque dos nutrientes contidos na serrapilheira acumulada, além de dar suporte para se entender as estratégias usadas pela vegetação na manutenção da sustentabilidade do ecossistema. Não é de se excluir nesse tipo de estudo a contribuição dada pelos organismos do solo na decomposição da matéria orgânica aportada ao solo. Isso ficou constatado no trabalho desenvolvido por Souto (2006) na RPPN Fazenda Tamanduá, em estudo desenvolvido no período de outubro de 2003 a setembro de 2005.

$\mathrm{O}$ autor acima afirma que a menor produção da fração folhas no período onde ocorrem maiores precipitações na Caatinga deve-se ao fato de que há uma renovação da folhagem, favorecida pela ocorrência das chuvas. Este fato observado por Nascimento et al. (2013) ao quantificarem a produção de serrapilheira em diferentes áreas sob fragmentos do Parque Nacional Serra de Itabaiana, Sergipe. Os autores ressaltaram que a redução da precipitação somada à redução do fotoperíodo, a formação florestal sofre estresse hídrico na seca, respondendo com maior queda das folhas, proporcionando maior produção de serrapilheira.
Diogo et al. (2014) ao analisarem a influência dos fatores abióticos na deposição de serapilheira na Reserva Natural da Serra das Almas, em Creteús, região centro-oeste do estado do Ceará, utilizando coletores de $0,5 \mathrm{~m}$ x $0,5 \mathrm{~m}$ durante doze meses (junho/2009 - maio/2010), verificaram uma produção total de $2.731,6 \mathrm{~kg} \mathrm{ha}^{-1}$ de serrapilheira depositada, sendo que, deste total a fração folha contribuiu com 54,3\%, estando o mês de agosto responsável pela maior deposição desse material.

Utilizando metodologia semelhante ao desta pesquisa Silva et al. (2015) ao quantificarem a deposição de serrapilheira em remanescente de caatinga no município de Cajazeirinhas, Paraíba, no período de dez meses (agosto/2013 - julho/2014) obtiveram valores de $1.630,5 \mathrm{~kg} \mathrm{ha}^{-1}$ de serrapilheira depositada sobre a superfície do solo, estando os meses de setembro de 2013 e junho de 2014 os maiores índices de deposição. Os autores verificaram ainda que, a quantidade de folha coletada nesse período foi superior à quantidade de galhos, e esta foi superior à quantidade de estrutura reprodutiva e miscelânea, sendo este comportamento semelhante a esta pesquisa.

Objetivando avaliar a contribuição do Croton blanchetianus Baill na produção de serrapilheira e ciclagem de nutrientes em área do Seridó da Paraíba no período de janeiro a novembro de 2011, Alves et al. (2014) verificaram uma produção de $1.207,91 \mathrm{~kg} \mathrm{ha}^{-1}$ de serrapilheira, 
verificando predominância da fração foliar $(79,78 \%)$ do material decíduo, seguida da fração galhos e cascas $(8,63 \%)$, estruturas reprodutivas $(5,92 \%)$ e fração miscelânea $(5,67 \%)$.

Santos et al. (2011), ao realizarem uma avaliação sazonal do aporte da serrapilheira em área de caatinga antropizada e em processo de regeneração natural na Estação Experimental do Instituto Agronômico de Pernambuco - IPA em Caruaru, observaram valor total de material depositado de $9.158,93 \mathrm{~kg}$ ha $\mathrm{ano}^{-1}$, valor este, superior comparado ao desta pesquisa. Os autores verificaram que a fração miscelânea, estimada $3.419,40 \mathrm{~kg}$ ha $\mathrm{ano}^{-1}$, representou $37,33 \%$ da serrapilheira, diferenciando dos resultados desta pesquisa e dos trabalhos citados acima.

Apesar dos resultados obtidos por Santos et al. (2011) com relação a uma maior produção de miscelânea, a fração foliar parece ser predominante na constituição da serrapilheira sobre a superfície do solo.

Representando a segunda maior produção do componente serrapilheira, a fração galhos + cascas mostrou-se sazonal durante o período experimental, chegando a totalizar 892,17 $\mathrm{kg} \mathrm{ha}^{-1}$, representando $21,2 \%$ do material aportado sobre a superfície do solo, ocorrendo concentração na deposição entre maio/2011 e fevereiro/2012 coincidindo boa parte desta deposição com o início do período seco e começo do período chuvoso (Tabela 1). Nota-se que houve variações no tocante à deposição de galhos + cascas durante o período de coleta, ou seja, de dezembro/2010 a agosto/2012. Neste trabalho foi verificada a queda de material lenhoso com diâmetro superior a 2,0 cm, o que significa que o material lenhoso coletado eram galhos finos, cascas e partes de galhos grossos que se desprendiam das árvores. Provavelmente pela ação mecânica do vento, a qual favoreceu a queda desses materiais.

Na Unidade de Conservação Estação Ecológica do Seridó, localizada no município de Serra Negra do Norte (RN), foi observado por Santana; Souto (2011) redução na produção da fração galhos + cascas à medida que as chuvas sessavam, com os menores picos de produção ocorrendo em novembrodezembro, quando choveu apenas $0,5 \mathrm{~mm}$ na área. Entretanto, com o início das chuvas, houve um substancial aumento na produção da fração, o que segundo os autores pode estar relacionado ao efeito mecânico da chuva no processo de deciduidade dos ramos ressequidos durante a época seca anterior.

Quanto à fração material reprodutivo (botões florais, flores, frutos e sementes) estimada em $605,58 \mathrm{~kg} \mathrm{ha}^{-1}$ houve sazonalidade no que se refere ao total armazenado nas caixas coletoras. Vê-se uma produção de material reprodutivo durante todo o ano (Tabela 2), não podendo se afirmar com tanta ênfase que a produção desse material está intrinsicamente ligado a fatores pluviométricos, visto que, mesmo nos períodos secos ou de baixíssimo índice pluviométrico, ocorreu queda desse material.

Tabela 2 - Coeficiente de decomposição (k), tempo médio de renovação $(1 / \mathrm{k})$ e tempos necessários para a decomposição de $50 \%$ e $95 \%$ da serrapilheira acumulada.

\begin{tabular}{ccccc}
\hline Período & $(\mathbf{k})$ & $\mathbf{1 / k}$ & $\begin{array}{c}\mathbf{t}_{\mathbf{0 , 5}} \\
(\mathbf{a n o s})\end{array}$ & $\begin{array}{c}\mathbf{t}_{\mathbf{0 , 0 5}} \\
(\mathbf{a n o s})\end{array}$ \\
\hline Dez/2010 - Ago/2012 & 1,36 & 0,73 & 0,94 & 2,20 \\
\hline
\end{tabular}

Segundo Santana; Souto (2011) há uma carência de trabalhos que quantifiquem a fração material reprodutivo; no entanto, os valores encontrados neste estudo evidenciam a necessidade de se monitorar, paralelamente, o comportamento fenológico das espécies ocorrentes nas áreas experimentais durante a fase das coletas. Devem-se coletar dados para que possam ajudar a explicar alguns resultados aparentemente contraditórios, e mesmo determinar a influência isolada de cada espécie para determinado evento ocorrido no processo de deposição. Foi observado que os materiais reprodutivos que mais contribuíram para os resultados foram os frutos de Angico, Imburana e a floração da Catingueira.

Com relação à fração miscelânea, sua produção durante o período de estudo foi estimado em $17,4 \mathrm{~kg} \mathrm{ha}{ }^{-1}$, correspondendo $0,41 \%$ da serrapilheira. A fração miscelânea é composta de fragmentos de folhas, galhos, ramos, flores, frutos, sementes e outros materiais vegetais de difícil identificação, além de insetos ou partes destes e fezes, apresentando desse modo grande variabilidade na sua composição e no seu padrão de deposição. Em alguns meses do período experimental, praticamente não ocorreu deposição dessa fração. Não se pode atribuir essa quase total inexistência de miscelânea à presença ou não de chuva, pois, por exemplo, em janeiro e fevereiro de 2011 ocorreram chuvas expressivas na área experimental e, não houve deposição dessa fração.

O coeficiente de decomposição obtido a partir da serrapilheira acumulada na RPPN Fazenda Tamanduá, no período de estudo foi de 1,36 (Tabela 2). Esses valores estão de acordo com Correia; Andrade (1999) para florestas tropicais de baixa altitude, variando de 1,1 a 3,3.

Os valores para $\mathrm{k}$ obtidos no presente estudo são similares aos encontrados por Souto (2006) na mesma área experimental. Isso indica que a mineralização da matéria orgânica será mais acelerada proporcionando uma maior liberação de nutrientes para a solução do solo. Já Santana (2005) encontrou para as condições do semiárido do Rio Grande do Norte valor k muito baixo $(0,33)$, demonstrando que a serrapilheira acumulada nessa área demora mais a mineralizar do que a encontrada na RPPN Fazenda Tamanduá.

Segundo Anderson e Swift (1983), o valor k tem sido amplamente utilizado para avaliar a taxa de decomposição da serrapilheira acumulada em uma determinada área. Algumas críticas têm sido atribuídas ao seu uso generalizado em ecossistemas que ainda não atingiram um equilíbrio entre o material que está sendo depositado no piso florestal e o material que é decomposto pelos microrganismos do solo.

Observando ainda a Tabela 3 , constata-se que o tempo médio de renovação $(1 / \mathrm{k})$ foi inferior a 1 ano, sendo semelhante ao encontrado por Souto (2006) na mesma área experimental e, inferior ao valor 3,03 encontrado por Santana (2005) na Caatinga da Estação Ecológica no Rio Grande do Norte.

O tempo necessário para a decomposição de $50 \%$ da serrapilheira acumulada é de aproximadamente 343 dias e, para decompor $95 \%$ da serrapilheira, o tempo é de 803 dias. Para a decomposição de $50 \%$ da serrapilheira acumulada o valor ora encontrado foi superior àquele obtido por Souto (2006) que foi de 0,63 e 0,49 para 01 ano e 02 anos, respectivamente. Já para a decomposição de $95 \%$, o valor obtido no presente estudo foi superior ao obtido por Souto (2006) na mesma área experimental $(2,11)$ para um período de 02 anos. 


\section{CONCLUSÕES}

A deposição da serrapilheira mostrou-se sazonal e ininterrupta durante o período de estudo, com a maior produção ocorrendo no início da estação seca e a menor nos meses finais da mesma estação;

A produção de serrapilheira obedeceu a seguinte ordem: folhas > galhos + cascas > material reprodutivo > miscelânea;

A serrapilheira acumulada no piso florestal pode ser decomposta em quase sua totalidade em aproximadamente dois anos.

\section{REFERÊNCIAS}

ALVES, G. S.; ALVES, G. F.; MARTINS, L. R. A.; SOUSA, J. S.; SOUTO, J. S. Contribuição do Croton blanchetianus Baill na produção de serrapilheira e ciclagem de nutrientes em área do Seridó da Paraíba. Revista Verde de Agroecologia e Desenvolvimento Sustentável, Pombal, v 9, n. 3, p. 50 - 57, 2014.

ANDERSON, J.M. £ SWIFT, M.J. 1983. Decomposition in tropical forests. In: Tropical Rain Forest: Ecology and Management. S.L. Sutton. T.C. Whitmores $£$ A.C. Chadwick (eds.). Special Publications Series of the British Ecological Society, London, n. 2, p. 287-309.

ANDERSON, J.N.; INGRAM, J.S.I. Tropical soil biology and fertility: A handbook of methods. Wallingford, $\mathrm{CAB}$ International, 1989. 171p.

ARATO, H.D.; MARTINS, S.V.; FERRARI, S.H. de S. Produção e decomposição de serrapilheira em um sistema agroflorestal implantado para recuperação de área degradada em Viçosa-MG. Revista Árvore, Viçosa, v. 27, n.5, p. 715721,2003

ARAÚJO, L. V. C. Composição florística, fitossociologia e influência dos solos na estrutura da vegetação em uma área de caatinga no semiárido paraibano. 2007, 111 f. (Tese). Universidade Federal da Paraíba.

CORREIA, M.E.F.; ANDRADE, A.G. Formação da serapilheira e ciclagem de nutrientes. In: SANTOS, G.A.; CAMARGO, F.A.O. (Eds.). Fundamentos da matéria orgânica do solo: ecossistemas tropicais e subtropicais. Porto alegre: Gênesis, 1999. p. 197-225.

COSTA, C. C. A.; CAMACHO, R. G. V.; MACEDO, I. D; SILVA, P. C. M. Análise comparativa da produção de serapilheira em fragmentos arbóreos e arbustivos em área de caatinga na FLONA de Açu-RN. Revista Árvore, Viçosa, v. 34, n. 2, p. 259-265, 2010.

DIOGO, I. J. S.; VIEIRA, I. R; SILVEIRA, A. P; ARAÚJO, F. S. The influence of abiotic factors on litter deposition in a semiarid area, northeastern Brazil. Rev. Inst. Flor. São Paulo, v. 26 n. 2 p. 203-213, 2014.

EMPRESA BRASILEIRA DE PESQUISA AGROPECUÁRIA. Centro Nacional de Pesquisas de Solos. Sistema brasileiro de classificação de solos. Rio de Janeiro, $2^{\mathrm{a}}$ ed., 2006. 306p.

KOLM, L; POGGIANI, F. Ciclagem de nutrientes em povoamentos de Eucalyptus grandis submetidos à prática de desbastes progressivos. Scientia Forestalis, Piracicaba, n.63, p. 79-93, 2003.
NASCIMENTO, A. F. J.; SILVA, T. O.; SAMPAIO, E. V. S. B.; FILHO, R. N. A.; DANTAS, T. V. P. Quantificação de serapilheira em diferentes áreas sob fragmentos do Parque Nacional Serra de Itabaiana, Sergipe. Semina: Ciências Agrárias, Londrina, v. 34, n. 6, p. 3271-3284, 2013.

OLSON, J.S. Energy storage and the balance of producers and decomposers in ecological systems. Ecology, Washington, v.44, n.2, p.322-331, 1963.

PAZ, J. H. A. Distribuição de indivíduos de três espécies arbóreas da caatinga provenientes da regeneração natural. 2010. 32 f. Monografia (Graduação em Engenharia Florestal) - Universidade Federal de Campina Grande, Centro de Saúde e Tecnologia Rural, Patos - PB.

QUEIROZ, A.F. Dinâmica da ciclagem de nutrientes contidos na serapilheira em um fragmento de mata ciliar no Estado de São Paulo. 1999. 93 f. Dissertação (Mestrado em Energia na Agricultura) - Faculdade de Ciências Agronômicas, Universidade Estadual Paulista, Botucatu, SP.

SANTANA, J. A. da S. Estrutura fitossociológica, produção de serapilheira e ciclagem de nutrientes em uma área de Caatinga no Seridó do Rio Grande do Norte. Tese. $184 \mathrm{f}$. (Doutorado em Agronomia) - Centro de Ciências Agrárias, Universidade Federal da Paraíba, Areia, PB. 2005.

SANTANA, J.A. da S.; SOUTO, J.S. Produção de serapilheira na Caatinga da região semiárida do Rio Grande do Norte, Brasil. Revista IDESIA, Arica, v. 29, n. 2, p. 87-94, 2011.

SANTOS, P. S.; SOUZA, J. T.; SANTOS, J. M. F. F.; SANTOS, D. M.; ARAÚJO, E. L. Diferenças sazonais no aporte de serrapilheira em uma área de caatinga em Pernambuco. Revista Caatinga, Mossoró, v. 24, n. 4, p. $94-$ 101, 2011.

SHANKS, R.; OLSON, J.S. First year breakdown of leaf litter in Southern Appalachian Forest. Science, Washington v.134, p. 194-195, 1961.

SILVA, V. N.; SOUTO, L. S.; FILHO, J. A. D.; SOUZA, T. M. A.; BORGES, C. H. A. Deposição de serapilheira em uma área de caatinga preservada no semiárido da Paraíba, Brasil. Revista Verde de Agroecologia e Desenvolvimento Sustentável, Pombal, v. 10, n.2, p. 21 - 25, 2015.

SOUTO, P. C. Acumulação e decomposição de serapilheira e distribuição de organismos edáficos em área de caatinga na Paraíba, Brasil. 2006. 150 f. Tese (Doutorado em Agronomia) - Universidade Federal da Paraíba, Areia, PB.

SOUTO, P. C.; SOUTO, J. S.; SANTOS, R. V.; BAKKE, I. A. Características químicas da serrapilheira depositada em área de caatinga. Revista Caatinga, Mossoró, v. 22, n. 1, 2009.

SOUTO, P. C.; SOUTO, J. S.; SANTOS, R. V.; BAKKE, I. A.; SALES, F. C. V.; SOUZA, B. V. Taxa de decomposição da serapilheira e atividade microbiana em área de caatinga. Cerne, Lavras, v. 19, n. 4, p. 559-565, 2013.

VITAL, A.R.T. Caracterização hidrológica e ciclagem de nutrientes em fragmento de mata ciliar em Botucatu, SP. 2002. Tese. 117 f. (Doutorado em Agronomia) - Faculdade de Ciências Agronômicas, Universidade Estadual Paulista, Botucatu, SP. 